\title{
MiR-455-3p regulates glioma cell proliferation by targeting PAX6
}

\author{
Jizhong Han, Yu Xiong, Huajiang Deng, Jie Zhou, Lilei Peng, Wei Xiang, Yang \\ Ming, Ligang Chen* \\ Department of Neurosurgery, Affiliated Hospital of Southwest Medical University, No. 25 Taiping Street, Jiangyang District, \\ Luzhou, Sichuan 646000, China
}

*For correspondence: Email: chenligangllg@163.com; Tel: 0086-0830-3165461

\begin{abstract}
Purpose: To investigate the role of miR-455-3p in gliomas.

Method: Quantitative real-time polymerase chain reaction was used to measure miR-455-3p and paired box 6 (PAX6) levels in glioma cell lines. Western blot analysis was used to determine the expression of cell cycle regulators. In addition to over-expression, silencing of miR-455-3p or PAX6 was performed to study the functions of miR-455-3p in gliomas.

Results: The levels of miR-455-3p were significantly up-regulated in glioma cell lines $(p<0.05)$, while miR-455-3p over-expression increased glioma cell proliferation and interfered with the progress of the cell cycle $(p<0.01)$. Furthermore, endogenous miR-455-3p silencing prevented glioma cell proliferation by regulating cell cycle progression $(p<0.05)$. The results also showed that PAX6 controlled the cell cycle while PAX6 silencing selectively regulated $p 21$ expression $(p<0.01)$. Furthermore, miR-455-3p and PAX6 influenced p53 expression. Re-introduction of PAX6 expressing vector into glioma cells rescued the pro-tumoral effect of miR-455-3p overexpression.

Conclusion: These findings demonstrate the role of miR-455-3p as a tumour oncogene in gliomas via regulation of the cell cycle, indicating that miR-455-3p might act as a new treatment strategy for glioma cell tumours and a predictor of survival in glioma patients.
\end{abstract}

Keywords: Glioma; Endogenous miR-455-3p, PAX6, Therapeutic target

This is an Open Access article that uses a fund-ing model which does not charge readers or their institutions for access and distributed under the terms of the Creative Commons Attribution License (http://creativecommons.org/licenses/by/4.0) and the Budapest Open Access Initiative (http://www.budapestopenaccessinitiative.org/read), which permit unrestricted use, distribution, and reproduction in any medium, provided the original work is properly credited.

Tropical Journal of Pharmaceutical Research is indexed by Science Citation Index (SciSearch), Scopus, International Pharmaceutical Abstract, Chemical Abstracts, Embase, Index Copernicus, EBSCO, African Index Medicus, JournalSeek, Journal Citation Reports/Science Edition, Directory of Open Access Journals (DOAJ), African Journal Online, Bioline International, Open-J-Gate and Pharmacy Abstracts

\section{INTRODUCTION}

Glioma is a type of malignant tumour of the central nervous system (CNS) that includes four clinical grades that are based on histology and prognosis $[1,2]$. Due to the inefficiency of current therapeutic approaches such as surgery, chemotherapy, and radiation [3], patients with gliomas have a median survival time of only 15 months and a low average survival rate of 5 years [4,5]. Although the effects of treatment on symptoms have improved in recent years, traditional treatment approaches for gliomas are usually invasive and have poor outcomes. Thus, it is necessary to find new intervention and treatment choices for glioma patients. Recent efforts have focused on new therapy strategies to 
modify the expression of tumourigenic genes or biochemical signalling pathways [6].

MicroRNAs (miRNAs) comprise a large family of endogenous noncoding RNA molecules with approximately 20 nucleotides [2]. These molecules cause translation inhibition or mRNA degradation, which result in biological effects in the body at the transcriptional and posttranscriptional levels via binding to the 3'untranslated region (3'-UTR) of target mRNAs [7]. Owing to their ability to modulate different target genes, miRNAs have crucial roles in diverse cellular behaviours in tumours, including proliferation, apoptosis, invasion, migration, and even glucose uptake [7]. Abnormal expression of miRNAs is reportedly related to tumour progression, and up-regulation or downregulation of miRNA leads to oncogenic and suppressive effects in tumours including lung cancer, gastric cancer, breast cancer, colorectal cancer, and even glioblastoma [8-10]. Moreover, miRNAs are a kind of diagnostic and prognostic index of disease progression [1].

Previous studies found that the abnormal expression of miR-455-3p may be closely associated with temozolomide (TMZ) resistance in the treatment of glioblastoma multiforme (GBM) [11,12]. A miRNA profiling analysis report shows that the expression of 30 miRNAs changes in the presence of GBM [13]. Seventeen miRNAs are down-regulated in migrating tumour cells. Among the most deregulated miRNAs, miR-455-3p is markedly up-regulated [13]. The expression pattern, biological functions, and potential molecular mechanism of miR-455-3p in gliomas are still unknown.

\section{EXPERIMENTAL}

\section{Cell lines}

Human glioma cell lines (SW1783, A172, U87, U251, and H4) and normal human astrocytes (NHA) cell lines were procured from the American Type Culture Collection (ATCC). The cell lines were cultured in modified medium containing $10 \%$ fetal bovine serum and penicillin-streptomycin (Gibco, Grand Island, NY).

\section{Quantitative real-time PCR (qRT-PCR)}

TRIzol Reagent (Tiangen, China) was used to extract RNA. Then, total RNA $(1 \mu \mathrm{g})$ procured using DNase (Life Technologies, Carlsbad, CA), was reverse-transcribed to cDNA by the Kit (Tiangen, China). The qRT-PCR was performed using the SYBR Green PCR Mix (Tiangen,
China). The PCR parameters were: $10 \mathrm{~min}$ at 95 ${ }^{\circ} \mathrm{C}$, followed by 40 cycles of $15 \mathrm{~s}$ at $95^{\circ} \mathrm{C}$ and 15 $\mathrm{s}$ at $60^{\circ} \mathrm{C}$. GAPDH was used as an endogenous control, and the U6 level was used as the miRNA control. The primer sequences are shown in Table 1.

Table 1: Primer sequence for the study

\begin{tabular}{ll}
\hline Primer & Sequence (5' $\left.\rightarrow \mathbf{3}^{\prime}\right)$ \\
\hline GAPDH-F & TGGACTCCACGACGTACTCAG \\
GAPDH-R & CGGGAAGCTTGTCATCAATGGAA \\
U6-F & CTCGCTTCGGCAGCACA \\
U6-R & AACGCTTCACGAATTTGCGT \\
PAX6-F & TGGGCAGGTATTACGAGACTG \\
PAX6-R & ACTCCCGCTTATACTGGGCTA \\
\hline
\end{tabular}

\section{Plasmids and siRNA transfections}

A lentiviral vector with miR-455-3p was constructed. The antisense oligonucleotides of miR-455-3p and ASO-NC (no silencing) were supplied by Sangon Co. (Shanghai, China). The siRNA of PAX6 and scrambles (control) were obtained from GenePharma Ltd. (Shanghai, China). The 3'-UTR of PAX6 was amplified and mutations of 3'-UTR of PAX6 were performed using a mutagenesis kit (Tiangen, China). The fragments were then cloned into luciferase reporter vector (Promega, USA). Subsequently, the vectors were transfected into cells using $X$ tremeGENE (Roche).

Cells were collected and lysed $48 \mathrm{~h}$ after transfection for the luciferase activity assay. Luciferase activity was measured using a dualluciferase reporter assay system (E1910, Promega, USA) according to the manufacturer's instructions.

\section{Western blotting}

Total cell proteins were subjected to $15 \%$ SDSPAGE, and then transferred onto polyvinylidene difluoride (PVDF) membranes. Next, membranes were blocked with $5 \%$ non-fat-dried milk. Primary antibodies were added to all membranes and incubated overnight. After incubating with corresponding secondary antibodies (Life Technology), the membranes were scanned. $\beta$ Actin was used as an internal control.

\section{MTT assay}

U87 and U251 cells were seeded in 96-well plates and subsequently transfected with $100 \mathrm{nM}$ of NC (no silencing), miR-455-3p, or ASO-miR455-3p. After $48 \mathrm{~h}$, MTT reagent was added to each well that contained $100 \mathrm{~mL}$ of medium that had been cultured for 3 days. Following 
incubation at $37{ }^{\circ} \mathrm{C}$ with a humidified $5 \% \mathrm{CO}_{2}$ for $2 \mathrm{~h}$, the well absorbance at $490 \mathrm{~nm}$ was tested.

\section{Cell cycle assay}

Cells $\left(1 \times 10^{6} /\right.$ well $)$ in a 6 -well plate were transfected with $100 \mathrm{nM}$ of expressing plasmids. After $48 \mathrm{~h}$ of culture, the cells were collected and washed three times with PBS. After fixing with ice-cold $70 \%$ ethanol, the fixed cells were incubated in a mixture of $0.5 \%$ Triton X-100, 1 $\mathrm{mg} / \mathrm{mL}$ RNase A, and $50 \mu \mathrm{g} / \mathrm{mL}$ propidium iodide (Becton Dickinson and Company, Franklin Lakes, NJ, USA) in PBS at $37{ }^{\circ} \mathrm{C}$ for $1 \mathrm{~h}$. Cell cycle was assessed using flow cytometry.

\section{Statistical analysis}

Data are expressed as mean \pm SEM. Statistical analysis was carried out with SPSS software (version 20.0, Chicago, IL, USA). Statistical differences among groups were compared using a Student's unpaired t-test following the one-way ANOVA method. Values of $p<0.05$ were regarded as statistically significant. Statistical analysis was performed using SPSS 20.0.

\section{RESULTS}

\section{MiR-455-3p was enhanced in glioma cells}

MiR-455-3p level in five glioma cell lines was evaluated using qRT-PCR. The miR-455-3p levels in glioma cell lines A172, SW1783, U87, $\mathrm{U} 251$, and $\mathrm{H} 4$ was increased compared to normal human astrocytes (NHAS) (Figure 1). This finding demonstrated that miR-455-3p level was markedly up-regulated in glioma cell lines.

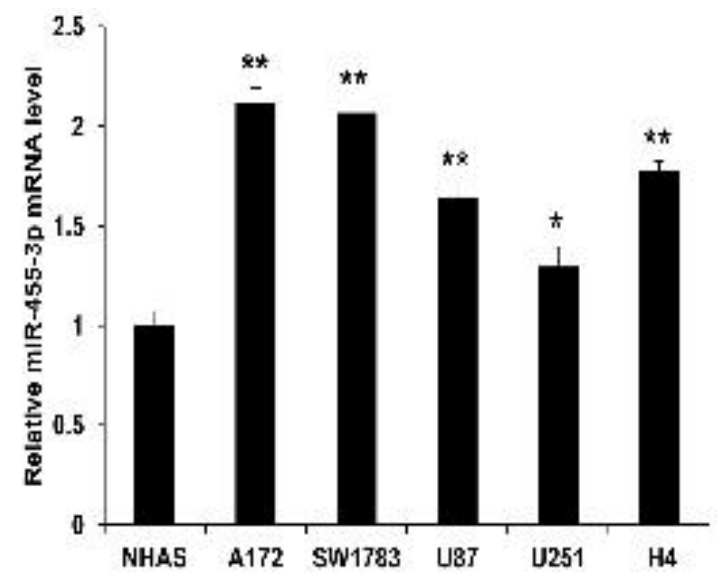

Figure 1: MiR-455-3p expression is enhanced in glioma cells. Relative miR-455-3p level in normal human astrocyte (NHAS) and glioma cell lines was evaluated by qRT-PCR; ${ }^{*} p<0.05$; ${ }^{* *} p<0.01$

\section{MiR-455-3p silencing suppressed cell proliferation}

To explore the roles of miR-455-3p in cell proliferation, U87 and U251 cells were transfected with ASO-NC, ASO-miR-455-3p, NC vector, or miR-455-3p overexpressing plasmids. Results of qRT-PCR showed an approximately six-fold increase miR-455-3p expression in cells transfected with overexpression plasmids. The miR-455-3p level was significantly decreased in the cells transfected with ASO-miR-455-3p when compared with those transfected with ASO-NC (Figure 2 A). MTT assays demonstrated that miR-455-3p overexpression markedly induced glioma cell proliferation, and that miR-455-3p suppression reversed this effect (Figure 2 B). These results indicate that overexpression of miR-455-3p might promote the proliferation of glioma cells.
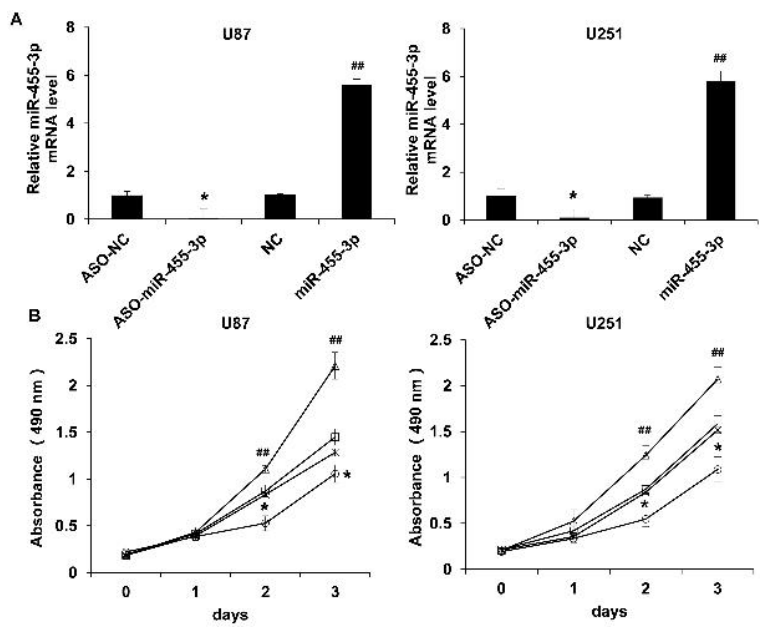

Figure 2: MiR-455-3p promotes glioma cell proliferation. (A) miR-455-3p level was evaluated in U87 and U251 cells by qRT-PCR. (B) MTT assay was performed to evaluate the proliferation of U87 and U251 cells; * $p<0.05$ compared with the ASO-NC group; $\left({ }^{\# \#}\right) p<0.01$ compared with the NC vector: 口)ASO-NC; (о) ASO-miR-455-3p; (x) NC; $(\Delta)$ miR455-3p

\section{PAX6 is a target of miR-455-3p}

It is well known that miRNAs function by regulating their downstream target genes. Therefore, TargetScan was used to search for miR-455-3p targets. As predicted, PAX6 was found to be a target of miR-455-3p (Figure $3 \mathrm{~A}$ ). Western blot assay showed that PAX6 in glioma cell lines was markedly lower than that in astrocyte cell line (Figure $3 \mathrm{~B}$ ). To confirm the supposition that PAX6 is the target of miR-455$3 p$, both mRNA and protein levels of PAX6 were evaluated in the U87 and U251 cells with miR$455-3 p$ suppression or overexpression. The data reveal that PAX6 protein and mRNA levels were 
significantly increased/decreased in the cells with miR-455-3p suppression/overexpression (Figure 3 C and D). Dual luciferase reporter assay showed that after miR-455-3p overexpression in U87 and U251, the luciferase activity of the cells co-transfected with wild-type 3'-UTR of PAX6 (which have a miR-455-3p binding site), was decreased. This result was not observed in the mutant 3'-UTR of PAX6 or the ASO-miR-455-3p co-transfected group (Figure $3 \mathrm{E}$ ). These results indicate that PAX6 were one target of miR-455$3 p$.

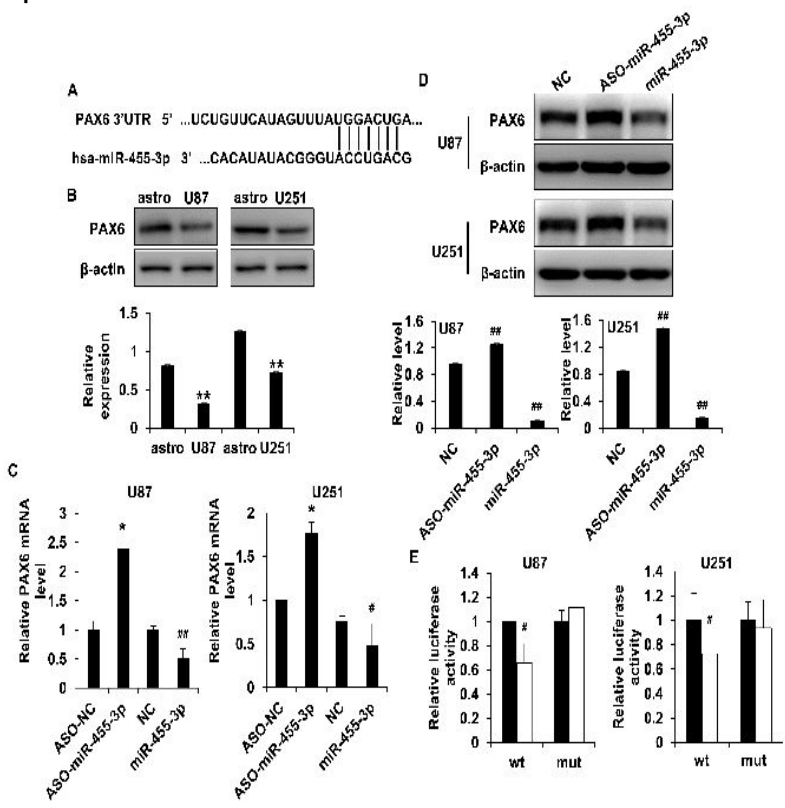

Figure 3: PAX6 is a target of miR-455-3p in glioma cells. (A) Sequence alignment between miR-455-3p and PAX6. (B) The expression of PAX6 in U87, U251, and astrocytic cells was evaluated by Western blot assay. (C and D) PAX6 level in U87 and U251 glioma cells was evaluated by qRT-PCR and Western blot. (E) Luciferase activity was performed in cells cotransfected with miR-455-3p and wild-type PAX6 3'UTR or the mutant PAX6 3'-UTR; $\mathbf{~ = ~ N C ; ~} \square=\mathrm{miR}$ 455-3p; ${ }^{*} p<0.05$ and ${ }^{* *} p<0.01$ compared between two groups $(\mathrm{B}) ;{ }^{*} p<0.05$, and ${ }^{* *} p<0.01$ compared to the ASO-NC; $\left({ }^{*} p<0.05\right.$ and ${ }^{\# \#} p<0.01$ compared with $\mathrm{NC}$ vector $(\mathrm{C}, \mathrm{D}$ and $\mathrm{E})$

\section{PAX6 regulates the expression of cell cycle- associated proteins in glioma cells}

To determine the function of PAX6 on cell cycle in glioma cell lines, PAX6 was knocked down using chemically synthesized PAX6-targeting siRNA (si-PAX6). As expected, results showed si-PAX6-transfected glioma cells had a much lower PAX6 level (Figure 4 A). When treated with si-PAX6, a marked increase in cell proliferation trend was detected in two glioma cell lines (Figure $4 \mathrm{~B}$ ). The effects of PAX6 silence on cell cycle circuitry detected in glioma cell lines were due to down-regulation of PAX6. Expression levels of Cyclin D1 (CCND1), Cyclin E2 (CCNE2), cyclin-dependent kinases (CDK2, CDK4, and CDK6), and P21 were evaluated in si-PAX6 transfected cells.

The above molecules have been proven to be key modulators of $\mathrm{G} 1$ phase [14]. As observed in Figure $4 \mathrm{C}$ and Figure $4 \mathrm{D}$, PAX6 silencing markedly enhanced CDK2, CDK4, CDK6, and cyclin D1 expressions in U87 and U251 cells, but P21 expression was decreased. These results suggest that PAX6 silence caused the elevation of the negative cell cycle regulators and downregulation of PAX6 promoted glioma cell proliferation. The E2F/DP1 transcriptional activity was also investigated. Paired box 6 downregulation led to hyper-phosphorylation of p130 (Figure $4 \mathrm{E}$ and Figure $4 \mathrm{~F}$ ). Moreover, cyclin/CDK complex activation induced the phosphorylation of $\mathrm{p} 130$, the elevated expression of dominant-negative regulators (DPs), and the enhanced E2F transcriptional activity (E2F/DP1 transcriptional activity), leading to the loss of tumour suppressor activity. These findings were further confirmed by the upregulated expression of E2F1, MDM2, and E2F5 (Figure $4 \mathrm{E}$ and Figure $4 \mathrm{~F}$ ).
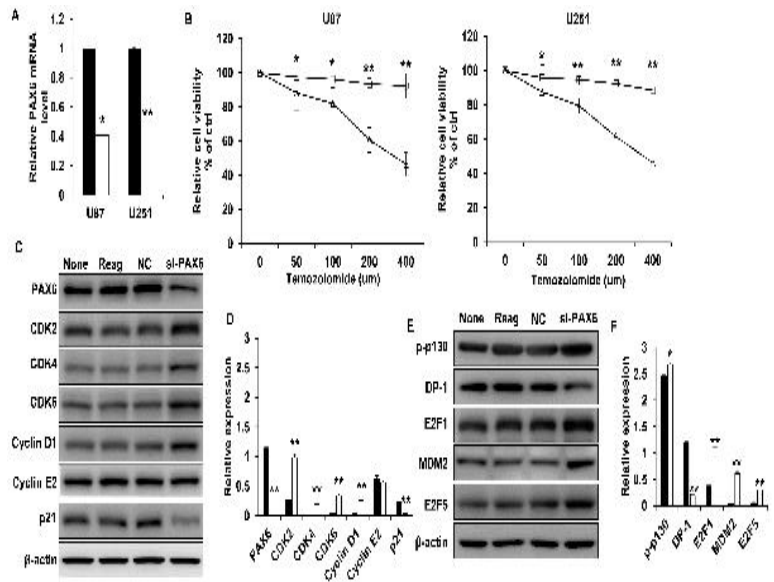

Figure 4: PAX6 inhibits the expression of regulatory components in G1/S cell cycle circuity. (A) The expression of PAX6 in U87 and U251 cells transfected with NC or si-PAX6 was evaluated by qRT-PCR; $\mathbf{\square}=$ NC; $\square=$ si-PAX6. (B) Cell proliferation was examined in U87 and U251 cells transfected with NC or si-PAX6 by MTT assay. $(\Delta)$ NC; ( $\square$ ) si-PAX6. (C) Western blot detected protein. The levels of CDK2, CDK4, CDK6, CCND1, CCNE2, and P21 in U87 and U251 cells was evaluated by Western blot. (D) Protein quantification results for $(C)$. ( $\left.{ }^{\square}\right) \mathrm{NC}$; $\left(^{\square}\right)$ si-PAX6. (E) Western blot detected protein The levels of p-130, DP-1, E2F1, and E2F5, and MDM2 in U87 and U251 cells was evaluated by Western blot. (F) Protein quantification results for (E). Key: $\square=\mathrm{NC} ; \square=$ si-PAX6; ${ }^{*} p<0.05$ and ${ }^{* *} p<0.01$ compared with the control 
Up-regulation of PAX6 reverse miR-455-3p overexpression effects on glioma

To further confirm that miR-455-3p exerted its function by directly targeting PAX6 in the glioma cell lines, miR-455-3p expressing plasmid was transfected together with a PAX6 expressing vector into the two cell lines, followed by a series of functional assays. This finding suggested that miR-455-3p might suppress the p53 expression. First, qRT-PCR and Western blot assay were performed to evaluate whether wild-type p53 was involved in changes induced by miR-455-3p and PAX6 expressions (Figure $5 \mathrm{~A}$ ). In addition, reintroduction of PAX6 reversed the miR-455-3p overexpression effect on cell proliferation (Figure $5 \mathrm{~B})$.
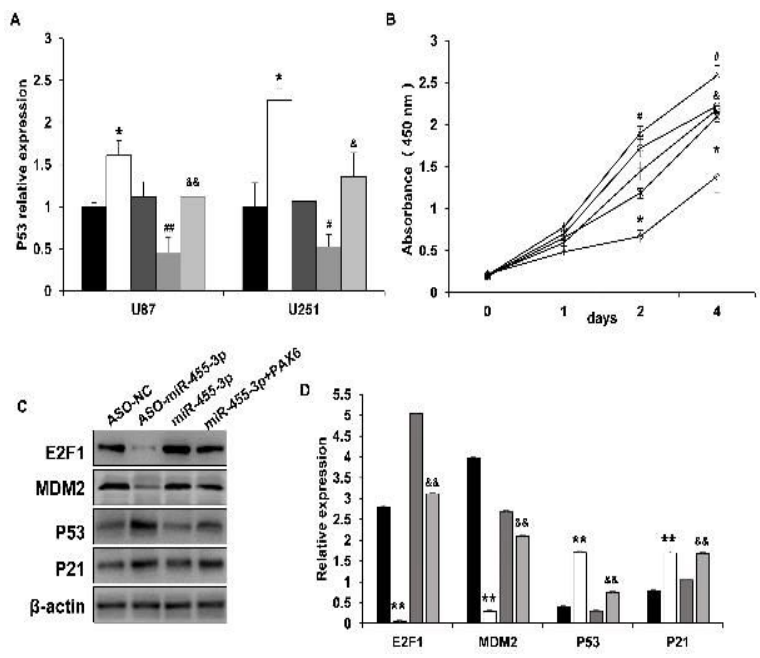

Figure 5: PAX6 reintroduction reversed the miR-455$3 p$-induced glioma cell growth. (A) Wild-type p53 level was detected in U87 and U251 cells with indicated vector transfection by qRT-PCR. Key: $\mathbf{m}=$ ASO-NC; $\square$ = ASO-miR-455-3p; = NC; ( $\square$ ) miR-455-3p; $(\square)$ miR-455-3p + PAX6. (B) Effects of PAX6 reintroduction on growth rates of U87 transfected with indicateayector were detected by MTT. Key: ( $\square$ ) ASONC; ( MSO-miR-455-3p; (×) NC; $(\Delta)$ miR-455-3p; (०) miR-455-3p + PAX6. (C and D) Protein levels of E2F1, MDM2, p53, and P21 in cells with indicated vector transfection were detected by Western blot. Key: $\mathbf{~ = ~}$ ASO-NC; $\square=$ ASO-miR-455-3p; $(\square)$ miR-455-3p; $(\square)$ miR-455-3p + PAX6; ${ }^{*} p<0.05$ and ${ }^{* *} p<0.01$ compared with ASO-NC; ${ }^{\#} p<0.05$ and ${ }^{\#} p<0.01$ compared with the NC vector; ${ }^{\&} p<0.05$ and ${ }^{\& \&} p<$ 0.01 compared with miR-455-3p overexpression group

In addition, co-transfection PAX6 overexpression vector with miR-455-3p overexpression vector abrogated the suppressed roles of miR-455-3p on E2F1 and MDM2, which were key molecules regulating cell cycle of glioma cells (Figures $5 \mathrm{C}$ and D). Furthermore, p53 and P21 expression levels also recovered following introduction of miR-455-3p-in (Figure $5 \mathrm{C}$ and Figure $5 \mathrm{D}$ ). All results suggest that PAX6 was verified as an object of miR-455-3p in glioma cells. Paired box 6 inhibition caused elevated expression levels of cyclin D1, CDK2, CDK4, and CDK6 in the G1/S transition of glioma cells. Furthermore, activated cyclin/CDK complex increased the expressions of E2F1 and MDM2, thus losing p53 tumour suppressor activity.

\section{DISCUSSION}

Glioma is one of the most malignant brain tumours and is common in adults $[15,16]$. Gliomas possess a subpopulation of selfrenewing cells and cancer stem cells that reproduce even in a poor tumour microenvironment [17]. Currently, therapies targeting cancer stem cells have become popular in the glioma treatment [18]. A growing number of studies have shown that miRNAs and IncRNAs are involved in the occurrence and progression of tumours. These findings may provide promising therapeutic strategies for gliomas.

The miRNAs up-regulated in gliomas always act as oncogenes that exert their impact on cell proliferation through regulating cell cycle systems $[19,20]$. Previous studies found up-regulated miR-455-3p levels in migrating GBM cells, which could result in elevated migration in vitro [13]. Another study found that miR-455-3p levels are markedly related to cell cycle and invasion in GBM, and miR-455-3pis involved in TMZ resistance in cancer stem cells (+) of GBM patients, providing a new therapeutic target for GBM treatment [11]. Herein, miR-455-3p and PAX6 levels in glioma cell lines were assessed and found to be deregulated in select glioma cell lines. This result implies that the deregulation of miR-455-3p might affect glioma initiation and development.

PAX6 is a member of the PAX family, which participates in encoding a group of paired-box transcription factors in various important bioactivities [21]. Paired box 6 plays a key role as a transcription factor in the development of the central nervous system [22]. Paired box 6 was recently demonstrated in multiple tumours, such as brain, pancreatic, and intestinal tumours [23]. The data show that suppression of PAX6 by miR$455-3 p$ led to an obvious increase in glioma cell proliferation. Luciferase assays proved that miR$455-3 p$ changed gene expression posttranscriptionally by binding to the 3'-UTR of PAX6. Down-expression of PAX6 facilitated cell proliferation, implying that PAX6 functions as an anti-oncogene in glioma. In addition, miR-455-3p and PAX6 co-overexpression reversed the 
promotion effect caused by miR-455-3p overexpression alone, revealing that miR-455-3p promoted glioma malignant behaviours via suppressing PAX6 expression. This result found that PAX6 is a miR-455-3p target in glioma cells. Using two glioma cell lines, this study further demonstrated that enhancement of miR-455-3p increased cell proliferation. In addition, PAX6 suppression elevated the expression of cyclins, such as cyclin D1, CDK2, and CDK4, in glioma cells. It was still unknown whether upregulation of these cyclins was induced by p21 directly regulated by PAX6. However, the regulation of cyclins and their functions in G1/S transition provided a role of PAX6 involvement in regulating cell cycle. It is possible that PAX6 deficiency in glioma cells resulted in hyperactivation of different cyclin/CDK complexes leading to an increase of DNA replication and even cell mitosis.

Previous studies have reported that p53 could regulate the expression of many miRNAs [24]. Moreover, almost all recent miRNAs studies indicate miRNAs are closely associated with p53 expression [15]. It has been reported that the TP53 tumour suppressor involved in human cancer, also function as a mediator in the tumourigenesis of glioma. p53 was reported to be targeted by miR-25 or miR-32, leading to the down-regulation of both protein and mRNA levels of p53 [25]. Research reports support the conclusion that p53 represses miRNAs through the transcriptional depression of E2F1 and other genes to affect the tumourigenesis process [15].

This study provides evidence of the oncogenic potential role of miR-455-3p in inducing cell growth. In addition, a network mechanism involving miR-455-3p directly co-regulating other factors in a p53-dependent manner through two transcriptional factors, E2F1 and MDM2, is described in this study. These results strongly suggest that PAX6 may function as a regulator controlling the expression level of CDK suppresser, CDKs, and cyclins in the G1/S transition. Taken together, these results bring to light the idea that the miR-455-3p- PAX6-p53 signalling plays a crucial role in human glioma.

\section{CONCLUSION}

This study shows that miR-455-3p increases cell proliferation in human glioma in vitro, and that microRNA-455-3p may function by directly targeting PAX6 and abnormal actions of PAX6, thereby disturbing homeostasis by dysregulating the cell cycle in glioma. Furthermore, the effect of abnormal regulation of miR-455-3p on cell cycle gene transcription plays a crucial role in the development of glioma. Further identification of the mechanism and function of miR-455-3p may contribute to understanding the effect of miRNAs on potential mitogenic functions in the development of glioma, thus providing new ideas for therapeutic targets.

\section{DECLARATIONS}

\section{Acknowledgement}

This work was funded as part of a project entitled "The Science and Technology Support Project of Sichuan Province" (grant no. 2013SZZ002) and "The Science and Technology Cooperation Project of Luzhou and Southwest Medical University" (grant nos. 14ZC0071-LH09 and 2016LZXNYD-G03).

\section{Conflict of interest}

The authors declare that no conflict of interest is associated with this work.

\section{Contribution of authors}

We declare that this work was done by the authors named in this article and all liabilities pertaining to claims relating to the content of this article will be borne by the authors. Jizhong Han and Ligang Chen designed all the experiments and revised the paper. Yu Xiong, Huajiang Deng, Jie Zhou, and Lilei Peng performed the experiments, Wei Xiang and Yang Ming wrote the manuscript.

\section{Open Access}

This is an Open Access article that uses a funding model which does not charge readers or their institutions for access and distributed under the terms of the Creative Commons Attribution License (http://creativecommons.org/licenses/by/ 4.0) and the Budapest Open Access Initiative (http://www.budapestopenaccessinitiative.org/rea d), which permit unrestricted use, distribution, and reproduction in any medium, provided the original work is properly credited.

\section{REFERENCES}

1. Shi Y, Tao T, Liu N, Luan W, Qian J, Li R, Hu Q, Wei Y, Zhang J, You Y. PPARalpha, a predictor of patient survival in glioma, inhibits cell growth through the E2F1/miR-19a feedback loop. Oncotarget 2016; 7(51): 84623-84633. 
2. Liu L, Cui S, Zhang R, Shi Y, Luo L. MiR-421 inhibits the malignant phenotype in glioma by directly targeting MEF2D. Am J Cancer Res 2017; 7(4): 857-868.

3. Demuth T, Berens ME. Molecular mechanisms of glioma cell migration and invasion. J Neurooncol 2004; 70(2): 217-228.

4. McLendon RE, Halperin EC. Is the long-term survival of patients with intracranial glioblastoma multiforme overstated? Cancer 2003; 98(8): 1745-1748.

5. Stupp R, Mason WP, van den Bent MJ, Weller M, Fisher B, Taphoorn MJ, Belanger K, Brandes AA, Marosi C, Bogdahn $U$ et al. Radiotherapy plus concomitant and adjuvant temozolomide for glioblastoma. N Engl J Med 2005; 352(10): 987-996.

6. Shi Y, Wang Y, Luan W, Wang P, Tao T, Zhang J, Qian J, Liu N, You Y. Long non-coding RNA H19 promotes glioma cell invasion by deriving miR-675. PLoS One 2014; 9(1): e86295.

7. Jiang K, Zhi T, Xu W, Xu X, Wu W, Yu T, Nie E, Zhou X, Bao Z, Jin $X$ et al. MicroRNA-1468-5p inhibits glioma cell proliferation and induces cell cycle arrest by targeting RRM1. Am J Cancer Res 2017; 7(4): 784-800.

8. Lee YS, Dutta A. MicroRNAs in cancer. Annu Rev Pathol 2009; 4: 199-227.

9. Moller HG, Rasmussen AP, Andersen HH, Johnsen KB, Henriksen M, Duroux M. A systematic review of microRNA in glioblastoma multiforme: micro-modulators in the mesenchymal mode of migration and invasion. Mol Neurobiol 2013; 47(1): 131-144.

10. Zhang Y, Dai G. Overexpression of MicroRNA-9 inhibits proliferation of human breast cancer cells by targeting STAT3. Trop J Pharm Res 2018; 17(9): 1753-1758.

11. Tezcan G, Tunca B, Bekar A, Preusser M, Berghoff AS, Egeli U, Cecener G, Ricken G, Budak F, Taskapilioglu $M O$ et al. microRNA expression pattern modulates temozolomide response in GBM tumors with cancer stem cells. Cell Mol Neurobiol 2014; 34(5): 679-692.

12. Ujifuku K, Mitsutake N, Takakura S, Matsuse M, Saenko V, Suzuki K, Hayashi K, Matsuo T, Kamada K, Nagata I et al. miR-195, miR-455-3p and miR-10a( *) are implicated in acquired temozolomide resistance in glioblastoma multiforme cells. Cancer Lett 2010; 296(2): 241-248.

13. Munthe $S$, Halle $B$, Boldt HB, Christiansen $H$, Schmidt $S$, Kaimal V, Xu J, Zabludoff S, Mollenhauer J, Poulsen FR et al. Shift of microRNA profile upon glioma cell migration using patient-derived spheroids and serumfree conditions. J Neurooncol 2017; 132(1): 45-54.
14. Malumbres M, Barbacid M. Cell cycle, CDKs and cancer: a changing paradigm. Nat Rev Cancer 2009; 9(3): 153166.

15. Suh SS, Yoo JY, Nuovo GJ, Jeon YJ, Kim S, Lee TJ, Kim T, Bakacs A, Alder H, Kaur B et al. MicroRNAs/TP53 feedback circuitry in glioblastoma multiforme. Proc Natl Acad Sci U S A 2012; 109(14): 5316-5321.

16. Yan SY, Chen MM, Li GM, Wang YQ, Fan JG. MiR-32 induces cell proliferation, migration, and invasion in hepatocellular carcinoma by targeting PTEN. Tumour Biol 2015; 36(6): 4747-4755

17. Wu $W$, Yang J, Feng $X$, Wang $H$, Ye $S$, Yang $P$, Tan $W$, Wei G, Zhou Y. MicroRNA-32 (miR-32) regulates phosphatase and tensin homologue (PTEN) expression and promotes growth, migration, and invasion in colorectal carcinoma cells. Mol Cancer 2013; 12: 30-38.

18. Yang F, Wang W, Zhou C, Xi W, Yuan L, Chen X, Li Y, Yang A, Zhang J, Wang T. MiR-221/222 promote human glioma cell invasion and angiogenesis by targeting TIMP2. Tumour Biol 2015; 36(5): 3763-3773.

19. Chivukula RR, Mendell JT. Circular reasoning: microRNAs and cell-cycle control. Trends Biochem Sci 2008; 33(10): 474-481.

20. Wang $Y$, Blelloch $R$. Cell cycle regulation by microRNAs in stem cells. Results Probl Cell Differ 2011; 53: 459472.

21. Osumi N, Shinohara H, Numayama-Tsuruta K, Maekawa M. Concise review: Pax6 transcription factor contributes to both embryonic and adult neurogenesis as a multifunctional regulator. Stem Cells 2008; 26(7): 16631672.

22. Yoshizaki K, Osumi N. [Molecular mechanism and mental function of postnatal neurogenesis]. Brain Nerve 2010; 62(12): 1315-1322.

23. Muratovska A, Zhou C, He S, Goodyer P, Eccles MR. Paired-Box genes are frequently expressed in cancer and often required for cancer cell survival. Oncogene 2003; 22(39): 7989-7997.

24. Raver-Shapira N, Marciano E, Meiri E, Spector $Y$, Rosenfeld N, Moskovits N, Bentwich Z, Oren $M$. Transcriptional activation of miR-34a contributes to $p 53-$ mediated apoptosis. Mol Cell 2007; 26(5): 731-743.

25. Kumar M, Lu Z, Takwi AA, Chen W, Callander NS, Ramos KS, Young KH, Li Y. Negative regulation of the tumor suppressor p53 gene by microRNAs. Oncogene 2011; 30(7): 843-853. 